\title{
Correlated Atom-Probe Tomography and Transmission Electron Microscopy of Meteoritic Nanodiamonds
}

\author{
J. B. Lewis ${ }^{1}$, D. Isheim ${ }^{2}$, C. Floss ${ }^{1}$, T. L. Daulton ${ }^{1,3}$, D. N. Seidman ${ }^{2}$ \\ 1. Laboratory for Space Sciences, Physics Department, Washington University, St. Louis, MO, USA. \\ 2. Center for Atom-Probe Tomography, and Dept. of Materials Science and Engineering, Northwestern \\ University, Evanston, IL, USA. \\ 3. Institute of Materials Science and Engineering, Washington University, St. Louis, MO, USA.
}

Primitive chondritic meteorites contain high concentrations of $\sim 3 \mathrm{~nm}$ nanodiamonds (NDs) [1]. NDs are isolated from meteorites by acid dissolution, and these isolates exhibit isotopic anomalies in trace elements, notably Xe, which suggest at least some are presolar in origin [2]. The carbon isotopic composition would provide a better indication of origin, but ND isolates are not pure, containing particles of amorphous, sp2-bonded carbonaceous particles of potentially different origins than the NDs [3]. We are applying atom-probe tomography (APT) to measure the ${ }^{12} \mathrm{C} /{ }^{13} \mathrm{C}$ composition of individual NDs. To identify NDs, it is necessary to confirm the size, density, and structure of the particles in reconstructed APT nanotips [4-6]. Transmission electron microscopy (TEM) is proving increasingly useful for correlated studies of nanostructures and nanoparticles with APT [7-9]. We report the results of correlated TEM/APT studies on two samples containing carbonaceous acid residue from the meteorite Allende.

A droplet of ND-containing Allende residue suspended in deionized water was deposited on a $\mathrm{Pt}$ substrate, while ultrasonicating to disperse the particles. The resulting deposit was covered by another layer of sputter-deposited Pt. Focused Ion-Beam (FIB) milling was utilized to lift out sections of the multilayer and attach them to posts on a $\mathrm{Cu}$ TEM half-grid. Samples were FIB-sharpened into nanotips designated HG01-B and $-\mathrm{C}$, each with $\sim 20 \mathrm{~nm}$ apex radii, suitable for APT (Fig. 1a). These nanotips were studied by TEM using a JEOL 2100F instrument at Washington University. Following TEM, a 50 $\mathrm{nm}$ Ni-layer was sputter-deposited on the nanotips. APT was conducted using a LEAP 4000X Si at Northwestern University. The last few hundred-million atoms were field evaporated from the nanotips utilizing $\sim 10 \mathrm{kV}$ DC voltage and thermal activation from a base temperature of $60 \mathrm{~K}$ combined with 80 pJ pulses from a $355 \mathrm{~nm}$ laser. APT reconstructions gave 3D positions and mass-to-charge-state ratios of $\sim 50 \%$ of the atoms within the volume analyzed.

Conventional TEM bright-field and scanning HAADF images (Fig. 1b-d) show discrete, low-density features 1-10 $\mathrm{nm}$ in diameter. Images taken at tilt angles from 0-35 degrees demonstrate that these features are coplanar, consistent with NDs embedded in Pt. However, they are also consistent with amorphous $\mathrm{C}$ or voids in the acid residue/Pt interface. APT reconstructions reveal hydrocarbon contamination on nanotip HG01-C, deposited by the TEM beam (Fig. 1e). ND deposition layers were identified in reconstructions of nanotips HG01-B and $-\mathrm{C}$, distinguished from hydrocarbon contamination by location in the $\mathrm{Pt}$, planar orientation, and the presence of residual salts and acids from separation treatments. While the deposition layers are filled with $\mathrm{C}$ at significantly higher atomic density than the surrounding $\mathrm{Pt}$, no discrete features were observed with the size and density expected for NDs.

The features observed by TEM suggest three possibilities. They are either voids created as Pt sputtercoated a rough sample deposit, clumps of amorphous $\mathrm{C}$, or NDs that were undetected by APT. A thick 
acid residue may weaken the attachment of NDs to the Pt, leading to NDs being plucked from the nanotips mostly whole during field-evaporation. In all cases, the $\mathrm{C}$ in the reconstructed deposition layers most likely represents amorphous $\mathrm{C}$.

Based on the results of this study, we will target lower-density regions of the acid residue deposit for our next samples. Correlated TEM/APT of nanotips containing only isolated, nanodiamond-sized carbonaceous regions, as has been previously observed in some cases [4-6], should resolve the question of whether we are imaging voids or clumps of acid residue in the TEM, and minimize the deleterious effects of a thick deposit on APT reconstructions [10].

\section{References:}

[1] Daulton T. L. et al, Geochimica et Cosmochimica Acta 60 (1996), pp. 4853-4872.

[2] Lewis R. S. et al, Nature 326 (1987), pp. 160-162.

[3] Daulton T. L. et al, J. Quatern. Sci., submitted (2016).

[4] Heck P. R. et al, Meteoritics and Planetary Science 49(3) (2014), pp. 453-467.

[5] Isheim D. et al, Microscopy and Microanalysis 19(Suppl 2) (2013), CD974-CD975.

[6] Lewis J. B. et al, Ultramicroscopy 159 (2015), pp. 248-254.

[7] Baik S.-I. et al, Scripta Materialia 68(11) (2013), pp. 909-912.

[8] Gorman B. P. et al, Microscopy Today 16(4) (2008), pp. 42-47.

[9] Rout S. S. et al. Microscopy and Microanalysis 21(Suppl 3) (2015), pp. 1313-1314.

[10] This work is supported by NASA grants NNX14AP15H (J.B.L.) and NNX13AF53G (C.F.), NSFMRI DMR-0420532 and ONR-DURIP N00014-0400798, N00014-0610539, and N00014-0910781 (LEAP tomograph at NUCAPT), the Initiative for Sustainability and Energy at Northwestern University (Instrumentation at NUCAPT), and MRSEC NSF DMR-1121262 (NUCAPT, through Northwestern's Materials Research Center).

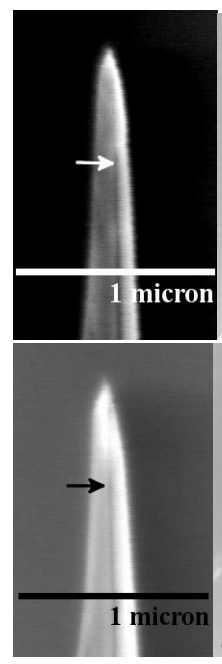

(a)

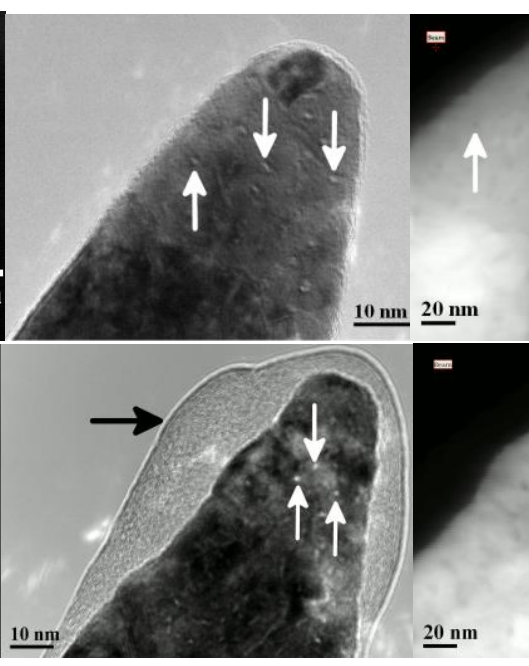

(b)
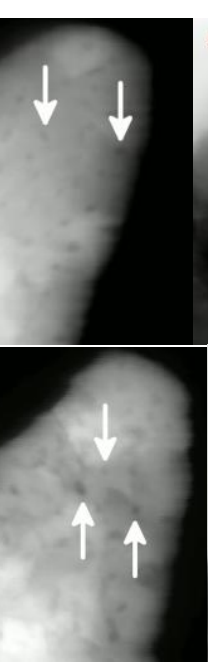

(c)

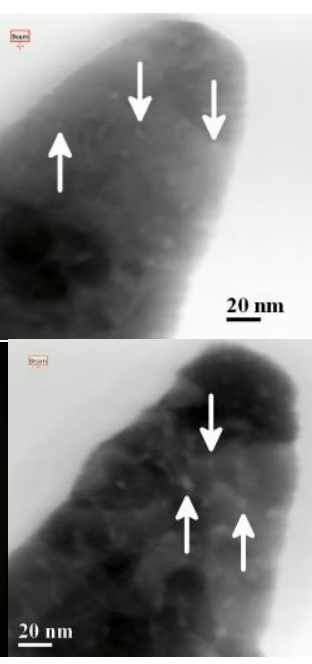

(d)

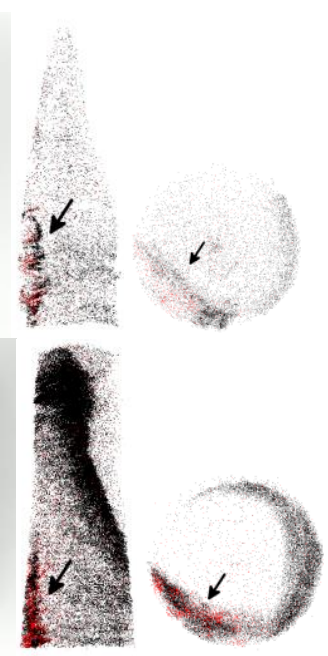

(e)

Figure 1: Secondary electron images of nanotips HG01-B (top row) and HG01-C (bottom row) prior to TEM analysis (a), showing the nanodiamond deposition layer (arrow) between Pt layers. TEM bright-field (b) as well as high angle annular dark field (c) and bright field (d) images show nm-sized low-density features, as well as hydrocarbon contamination on HG01-C. APT 3D maps (e) of carbon (black) and $\mathrm{NaO}$ (red) ions show the carbon-filled deposition layers, and, for HG01-C, the hydrocarbon cap. 